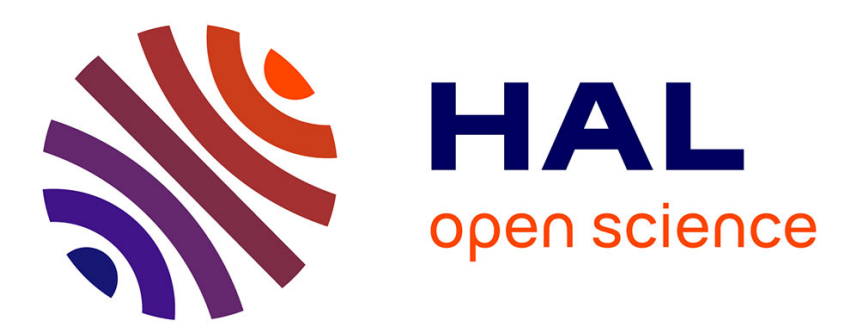

\title{
Modal Analysis of Chiral Metamaterial Using Characteristic Mode Analysis and Eigenmode Expansion Method
}

Nadia Kari, Divitha Seetharamdoo, Jean-Marc Laheurte, Francois Sarrazin

\section{- To cite this version:}

Nadia Kari, Divitha Seetharamdoo, Jean-Marc Laheurte, Francois Sarrazin. Modal Analysis of Chiral Metamaterial Using Characteristic Mode Analysis and Eigenmode Expansion Method. IEEE Journal of Multiscale and Multiphysics Computational Techniques, 2020, 5, pp.37-43. 10.1109/JMMCT.2020.2981235 . hal-02545823

\section{HAL Id: hal-02545823 \\ https://hal.science/hal-02545823}

Submitted on 1 Feb 2021

HAL is a multi-disciplinary open access archive for the deposit and dissemination of scientific research documents, whether they are published or not. The documents may come from teaching and research institutions in France or abroad, or from public or private research centers.
L'archive ouverte pluridisciplinaire HAL, est destinée au dépôt et à la diffusion de documents scientifiques de niveau recherche, publiés ou non, émanant des établissements d'enseignement et de recherche français ou étrangers, des laboratoires publics ou privés. 


\title{
Modal Analysis of Chiral Metamaterial Using Characteristic Mode Analysis and Eigenmode Expansion Method
}

\author{
Nadia Kari* ${ }^{* \dagger}$, Divitha Seetharamdoo*, Jean-Marc Laheurte ${ }^{\dagger}$, François Sarrazin ${ }^{\dagger}$
}

\begin{abstract}
A chiral metamaterial (MTM) is analyzed using two modal analysis methods: Characteristic Mode Analysis (CMA) and Eigenmode Expansion Method (EEM). Chiral MTMs are commonly associated to antennas and other microwave devices due to their ability to interact with electromagnetic waves and alter their polarizations. Here, the interaction of a chiral helix with linearly polarized electromagnetic plane waves is studied and asymmetric transmission is demonstrated. The different metrics given by CMA and EEM are analyzed and compared. It is shown that the modal weighing coefficient is a good metric to analysis the chiral properties of the MTM and these results are validated using a full-wave approach. It is also shown that if the two modal approaches propose very close results qualitatively, the maximum value of the modal current density obtained by EEM is closer to the one obtained by full-wave simulations. The modal approaches presented to analyse chiral MTM can be applied for the analysis of circularly polarised chiral metamaterial-inspired antennas.
\end{abstract}

\section{INTRODUCTION}

Over the last two decades, numerous research works have investigated Metamaterials (MTMs) which are highly desirable for applications ranging from antennas to microwave devices. The significant contribution of MTMs in improving antenna performances, such as gain [1], bandwidth and radiation pattern [2] has been widely demonstrated. In [3], MTM-inspired antennas include resonant parasitic elements into the nearfield region of a driven radiator so that the strong coupling between them helps to achieve high radiation efficiency. More recently, exotic electromagnetic properties of chiral MTMs have been experimentally demonstrated including negative index refraction [4], [5], elliptical or circular dichroism [6], polarization transformation [7] and giant optical activity [8]. Another appealing feature is the circular conversion dichroism yielding an asymmetric transmission phenomenon which can be of interest for the design of circularly polarized MTM antennas [9].

Calculation of the effective parameters (electric permittivity and magnetic permeability) [10], [11] is a common technique for the design and evaluation of MTMs. However, numerical techniques based on modal expansions such as the Eigenmode

*Univ Lille Nord de France, IFSTTAR, COSYS, LEOST, F59650 Villeneuve d’Ascq, France. nadia.kari@univ-paris-est.fr, divitha.seetharamdoo@ifsttar.fr

† Université Paris-Est, ESYCOM (FRE 2028), CNAM, CNRS, ESIEE Paris, F-77454 Marne-la-Vallée, France.

This paper is an expanded version of a conference paper presented at IEEE Int. Symposium on Antennas and Propagation, Atlanta, GA.
Expansion Method (EEM) [12] and Characteristic Mode Analysis (CMA) [13] are now extensively studied [14] to bring more physical insight on the radiation phenomena. EEM and CMA are attractive methodologies for MTM antenna designs because they are excitation independent and applicable to arbitrarily shaped structures. Furthermore, since it has been claimed [3] that enhanced antenna performances of electrically small MTM antennas are obtained by stored energy compensation, the evaluation of modal stored energies by MTMs is of great interest. Stored energy is based on the knowledge of the current sources of energy densities and on the evaluation of eigenvalues [15], [16]. The comparison between CMA and EEM is interesting since CMA is an approximate and fast approach with real eigenvalues and eigencurrents, whereas EEM is an absolute approach with complex eigenvalues and eigencurrents.

To date, chiral MTMs have been analysed using the numerical or analytical evaluation of reflection and transmission coefficients [17]. The purpose of this paper is to demonstrate that modal approaches can bring more physical insight for the design of efficient chiral antennas. To support this objective, it is essential to show which metrics of modal approaches are relevant to highlight the properties of chiral MTM and how to compare them to existing techniques [18]. In [19], an intrinsic chiral MTM based on a spiral helix was analyzed for the first time using both EEM and CMA. The net stored energy of the inclusion was evaluated and it was demonstrated that a circular polarized (CP) wave is transmitted by the helix when it is excited by a linearly polarized plane wave at normal incidence. In this expanded paper, we are more specifically interested in studying, interpreting and comparing the metrics given by both CMA and EEM methods for the chiral MTM helix. These metrics include stored energy, Modal Significance (MS), modal surface current density and Modal Weighting Coefficients (MWCs). The asymmetric transmission of the chiral helix is demonstrated using the MWCs and validated by a comparison with a full-wave analysis. A qualitative and quantitative analyses will also be proposed to highlight the most appropriate modal approach for studying this chiral MTM.

The paper is organized as follows. In section II, CMA and EEM are introduced and compared. Section III gives a brief review of the chiral MTM helix under consideration and details its properties such as the asymmetric transmission of linearly polarized EM waves. In section IV, results in terms of modal metrics are presented along with a numerical validation using 
a full-wave analysis. Section V finally discusses the relevance of the modal approaches for studying chiral MTM while proposing a critical comparison between the two approaches CMA and EEM.

\section{MODAL APPROACHES}

\section{A. Eigenmode Expansion Method (EEM)}

The eigenmodes are described by the standard eigenvalues equation [12], [20] as follows:

$$
Z\left(\vec{I}_{n}^{\text {eem }}(\vec{r}, \omega)\right)=\Upsilon_{n}^{\text {eem }} \vec{I}_{n}^{\text {eem }}(\vec{r}, \omega) .
$$

where $\mathrm{Z}$ is a linear operator, $\vec{I}_{n}^{\text {eem }}$ and $\Upsilon_{n}^{\text {eem }}$ are the eigencurrents and eigenvalues, respectively, $\tilde{\mathrm{r}}$ represents the field point and $\omega$ denotes the angular frequency. The EEM can be computed from a modal decomposition of the Method of Moments (MoM) impedance matrix [21]. It is obvious from (1) that the eigenvalues and the eigencurrents are independent from the incident field. Both $\Upsilon_{n}^{\text {eem }}$ and $\vec{I}_{n}^{\text {eem }}$ are complex quantities.

Let us consider a Perfect Electric Conductor (PEC) body excited with an incident field $\vec{E}^{i}$. The induced current $\vec{I}$ produced by the incident field radiates a scattered field $\vec{E}^{s}$. After applying the boundaries conditions on the tangential field, the scattered field can be defined by [21]:

$$
\left[\vec{E}^{s}\right]_{\text {tan }}=-Z\left(\vec{I}^{\mathrm{eem}}\right) \text {. }
$$

Equation (2) demonstrates that the tangential component of the electric field and the current distribution have the same behavior on the surface of PEC bodies. Applying the orthogonality, the total current is given by:

$$
\vec{I}^{\mathrm{eem}}=\sum_{n} \frac{\left\langle\vec{I}_{n}^{\mathrm{eem}}, \vec{E}^{i}\right\rangle}{\Upsilon_{n}^{\mathrm{eem}}\left\langle\overrightarrow{I_{n}^{\mathrm{eem}}}, \overrightarrow{\left.I_{n}^{\mathrm{eem}}\right\rangle}\right.} \vec{I}_{n}^{\text {eem }} .
$$

Using (1) and (2) and considering the Poynting theorem, the eigenmodes can be expressed as

$$
\Upsilon_{n}^{\text {eem }}=\frac{P_{\mathrm{rad}}\left(\vec{I}_{n}^{\mathrm{eem}}\right)+j \omega\left[W_{m}\left(\vec{I}_{n}^{\text {eem }}\right)-W_{e}\left(\vec{I}_{n}^{\text {eem }}\right)\right]}{\left\langle\vec{I}_{n}^{\text {eem } *}, \vec{I}_{n}^{\text {eem }}\right\rangle},
$$

where $P_{\text {rad }}\left(\vec{I}_{n}^{\text {eem }}\right)$ is the radiated power and $\left[W_{m}\left(\vec{I}_{n}^{\text {eem }}\right)-\right.$ $\left.W_{e}\left(\vec{I}_{n}^{\text {eem }}\right)\right]$ represents the net stored energy of the eigenmode. After normalizing (4) to $\left\langle\overrightarrow{I_{n}^{\text {eem }} *}, \overrightarrow{I_{n}^{\text {eem }}}\right\rangle$, the eigenvalues become:

$$
\Upsilon_{n}^{\text {eem }}=P_{\text {rad }}\left(\vec{I}_{n}^{\text {eem }}\right)+j \omega\left[W_{m}\left(\vec{I}_{n}^{\text {eem }}\right)-W_{e}\left(\vec{I}_{n}^{\text {eem }}\right)\right] .
$$

To easily manipulate the eigenvalues of the eigenmodes, which are complex quantities as defined above, it is important to define the ratio between the imaginary and real parts of the eigenvalues $\Upsilon_{n}^{e e m}$ [22], [23]. Using (5), we can introduce:

$$
\lambda_{n}^{\mathrm{eem}}=\frac{\left[W_{m}\left(\overrightarrow{I_{n}^{\mathrm{eem}}}\right)-W_{e}\left(\overrightarrow{I_{n}^{\mathrm{eem}}}\right)\right]}{P_{\mathrm{rad}}\left(\overrightarrow{I_{n}^{\mathrm{eem}}}\right)} .
$$

As $\lambda_{n}^{\text {eem }}$ represents the ratio between the net stored power and the radiated power, it gives some physical insight on the nature of the eigenmodes [24]. Therefore, for antennas and scattering problems, it is interesting to focus on the eigenmodes with the smallest magnitude of $\lambda_{n}^{\text {eem }}$.

\section{B. Characteristic Mode Analysis (CMA)}

Characteristic modes (CMs) can be defined with the generalized eigenvalues problem [14]:

$$
X\left(\vec{I}_{n}^{\text {cha }}(\vec{r}, \omega)\right)=\lambda_{n}^{\text {cha }} R\left(\vec{I}_{n}^{\text {cha }}(\vec{r}, \omega)\right),
$$

where $\lambda_{n}^{\text {cha }}$ are the characteristic values, $\vec{I}_{n}^{\text {cha }}$ are the characteristic currents, and $R$ and $X$ are respectively the real and imaginary parts of the impedance operator [21], $R=\frac{Z+Z^{*}}{2}$ and $X=\frac{Z-Z^{*}}{2 j}$ being symmetric and real matrices [14], [25]. Since $I_{n}^{\text {cha }}$ is a real quantity, the characteristic currents are equiphase. The characteristic values are defined by:

$$
\lambda_{n}^{\text {cha }}=\frac{\left[W_{m}\left(I_{n}^{\text {cha }}\right)-W_{e}\left(I_{n}^{\text {cha }}\right)\right]}{P_{\text {rad }}\left(I_{n}^{\text {cha }}\right)} .
$$

Based on the reciprocity theorem, it is known that if $Z$ is a linear symmetric operator then $R$ and $X$ are real symmetric operators. Therefore, the characteristic values $\lambda_{n}^{\text {cha }}$ and the characteristic currents $I_{n}^{\text {cha }}$ are real quantities. It is important to note that the characteristic values and currents are independent of any excitation. The characteristic currents $\vec{I}_{n}^{\text {cha }}$ on the PEC depend only on the conductor shape and size [20]. The total current $\vec{I}_{n}^{\text {cha }}$ can be defined as the combination of all modal currents:

$$
\vec{I}^{\mathrm{cha}}=\sum_{n=1}^{N} c_{n}{\overrightarrow{I_{n}^{\mathrm{cha}}}}^{\mathrm{cha}}
$$

where $c_{n}$ is the complex modal expansion coefficient which defines the weighting of each mode of the total current. The total current can also be written as:

$$
\vec{I}^{\text {cha }}=\sum_{n=1}^{N} \frac{V_{n}^{\mathrm{i}} \vec{I}_{n}^{\text {cha }}}{1+j \lambda_{n}^{\text {cha }}}
$$

where $V_{n}^{\mathrm{i}}$ represents the modal excitation coefficient. By combining (9) and (10), the modal expansion coefficients can be expressed as:

$$
\begin{gathered}
\left|c_{n}\right|=\left|\frac{V_{n}^{i}}{1+j \lambda_{n}^{\text {cha }}}\right|=\left|V_{n}^{\mathrm{i}}\right| \mathrm{MS}, \text { and } \\
\mathrm{MS}=\left|\frac{1}{1+j \lambda_{n}^{\text {cha }}}\right| .
\end{gathered}
$$

From (11), it is interesting to note that the excitation of the desired mode not only requires a large Modal Significance (MS) but also a large modal excitation coefficient. Moreover, to better define the coupling between the nth characteristic current and an external excitation, we define the MWCs as follows:

$$
W_{n}=\frac{V_{n}^{\mathrm{i}}}{1+j \lambda_{n}^{\text {cha }}} .
$$

The eigenvalues of the CMs represent the ratio between the net stored power and the radiated power. As for the EEM, the net stored energy within a scattering or radiation problem is proportional to the magnitude of the eigenvalues.

\section{Stored energy for CMA and EEM}

For both EEM and CMA, the eigenvalues give a physical insight in terms of net stored energy. Modes with positive 
eigenvalues are linked to magnetic stored energy, whereas modes with negative eigenvalues are linked to electric stored energy. Zero eigenvalues indicate a resonance. Also, the evaluation of the stored energy in the near-field allows to better classify the MTM as a magnetic or an electric structure, i.e., to better associate MTMs to antennas according to their electric or magnetic nature. The expression of the stored energy for the radiating structure was determined in [15]. Other approaches were proposed in [16], [26]. It is important to note that for EEM, the computation of the stored energy is based on the ratio between the imaginary and real parts of the eigenvalues, as mentioned in (6). In this paper, the evaluation of the stored energy $W_{\text {sto }}$ is based on the expression [27] given by:

$$
W_{\text {sto }}=\frac{1}{4 \omega} I^{H} X^{\prime} I,
$$

where $X^{\prime}$ is expressed in (13) by:

$$
X^{\prime}=\omega \frac{\partial X}{\partial \omega},
$$

$I$ represents the eigencurrents and the superscript $H$ denotes the Hermitian transpose. The stored energy $W_{\text {sto }}$ is the contribution of electric $\left(W_{\mathrm{e}}\right)$ and magnetic $\left(W_{\mathrm{m}}\right)$ energies. These quantities are defined as:

$$
\begin{gathered}
W_{\mathrm{m}}=\frac{1}{8 \omega} I^{H} X_{\mathrm{m}} I, \text { and } \\
W_{\mathrm{e}}=\frac{1}{8 \omega} I^{H} X_{\mathrm{e}} I,
\end{gathered}
$$

where $X$ is the reactance part of the impedance matrix and $X_{\mathrm{e}}=X^{\prime}-X$ and $X_{\mathrm{m}}=X^{\prime}+X$.

These equations are used both for CMA and EEM to compute the energy stored by complex structures. For CMA, the stored energies are real quantities and for EEM, the stored energies are complex quantities since the eigencurrents are complex values. The net stored energy is thus evaluated as $\Re e\left(W_{\mathrm{m}}-W_{\mathrm{e}}\right)$.

\section{Comparison between CMA and EEM}

The main features and properties of the metrics associated to the two modal approaches are summarised in Table I. CMA is an approximate approach since the characteristic values and currents are assumed real, i.e, the phase current is neglected, making the interpretation of CMs easier. A major feature that makes CMA attractive is that the CMs are orthogonal in terms of currents and far fields. Thus, the CMs form a very useful basis set in the expansion of any possible fields or currents associated with a PEC body. On the other hand, EEM is an absolute approach because the eigen values and currents are complex quantities. The EEM eigenmodes do not hold the electromagnetic power orthogonality in general since the eigenmodes are complex. In section IV, a comparison between the two methods is performed.

\section{DESCRIPTION OF CHIRAL METAMATERIAL}

In this section, a brief review of chirality is introduced. Chi-

\begin{tabular}{|c|c|}
\hline CMA & EEM \\
\hline $\begin{array}{l}\text { Based on the gen- } \\
\text { eralized eigenvalues } \\
X\left(\overrightarrow{I_{n}}{ }^{c h a}(r, \omega)\right)= \\
\lambda_{n}^{c h a} R\left({\overrightarrow{I_{n}}}^{c h a}(r, \omega)\right)\end{array}$ & $\begin{array}{l}\text { Based on the } \\
\text { standard eigenvalues } \\
Z\left(\overrightarrow{I_{n}}{ }^{e e m}(r, \omega)\right)= \\
\Upsilon_{n}^{e e m}{\overrightarrow{I_{n}}}^{e e m}\end{array}$ \\
\hline $\begin{array}{l}\text { Approximate } \\
\text { approach }\end{array}$ & Absolute approach \\
\hline $\begin{array}{l}\text { Easy to manipulate } \\
\text { (Real quantities) }\end{array}$ & $\begin{array}{l}\text { Difficult to manipu- } \\
\text { late (Complex quan- } \\
\text { tities) }\end{array}$ \\
\hline $\begin{array}{l}\text { The stored and the } \\
\text { net stored energies } \\
\text { are equal and real } \\
\text { quantities (real cur- } \\
\text { rents) }\end{array}$ & $\begin{array}{l}\text { The stored energy is } \\
\text { complex (complex } \\
\text { currents) and the net } \\
\text { stored energies is } \\
\text { evaluated as the real } \\
\text { part of the stored } \\
\text { energy }\end{array}$ \\
\hline \multicolumn{2}{|c|}{$\begin{array}{l}\text { The characteristic (eigen) values and current } \\
\text { are independent from the source. }\end{array}$} \\
\hline
\end{tabular}
ral MTMs represent a special category of artificial structures
TABLE I

COMPARISON OF PROPERTIES BETWEEN CMA AND EEM.

referring to geometries that cannot superpose to their mirror images and have no plane of symmetry. The electromagnetic properties of chiral material are Optical Activity (OA) and Circular Dichroism (CD). OA represents the rotation of a polarization wave after propagating through a chiral material. CD defines the absorption or transmission between the Left Hand Circularly Polarized (LHCP) and the Right Hand Circularly Polarized (RHCP) waves [28]. As a consequence of anti-symmetry in chiral MTMs, a high coupling between the magnetic and electric fields exists in this category of MTMs. The relationship equation between the electric and magnetic fields is described by:

$$
\vec{D}=\epsilon \vec{E}+j \kappa \vec{H}, \text { and }
$$

$$
\vec{B}=\mu \vec{H}+j \kappa \vec{E}
$$

where $\vec{D}, \vec{E}, \vec{H}, \vec{B}$ are the displacement vector, electric field, magnetic field and the magnetic flux density, respectively. The parameters $\epsilon, \mu$ and $\kappa$ are the permittivity, permeability and the chiral parameter, respectively. The latter defines the coupling strength between the magnetic and the electric fields. When $\eta$ is equal to zero, (18) and (19) have the same form as isotropic materials. In Table II, a comparison of the properties of a chiral medium and a simple isotropic medium is presented. It is interesting to note that the intrinsic wave impedance is independent of $\eta$. Due to the existence of $\eta$, the degeneracy of the two CP waves is broken, i.e., the refractive index is increased for one CP and reduced for the other one [29].

There are two types of chiral MTMs, the extrinsic chiral MTM, with a non-symmetry of the 3D structure and the intrinsic chiral MTM such as the chiral helix which has intrinsic chirality for its continuous stereo structure [28]. The latter type of chiral MTMs is widely used to achieve the 
TABLE II

COMPARISON OF PROPERTIES BETWEEN ISOTROPIC AND CHIRAL MEDIUM.

\begin{tabular}{|l|l|l|}
\hline Characteristics & $\begin{array}{l}\text { Isotropic } \\
\text { Medium }\end{array}$ & Chiral Medium \\
\hline \multirow{3}{*}{ Constitutive relations } & $\vec{D}=\epsilon \vec{E}$ & $\vec{D}=\epsilon \vec{E}+j \kappa \vec{H}$ \\
\hline Wave-numbers & $\vec{H}=\vec{B} / \mu$ & $\vec{B}=\mu \vec{H}+j \kappa \vec{E}$ \\
\hline Eigenmodes & $\omega \sqrt{\mu \epsilon}$ & $k_{ \pm}=\omega[ \pm \gamma+$ \\
& $\begin{array}{l}\text { Linear po- } \\
\text { larization }\end{array}$ & $\begin{array}{l}\text { Circular polariza- } \\
\text { tion }\end{array}$ \\
\hline Intrinsic impedance & $\zeta=\sqrt{\mu \epsilon}$ & $\zeta_{c}=\sqrt{\mu_{c} \epsilon_{c}}$ \\
\hline
\end{tabular}

broadband conversion of polarization in the terahertz range. In this paper, we are interested in the optimal chiral helix characterized by its equivalent magnetic, dielectric and chiral properties [18]. The equivalence between these three properties leads to a circularly polarized wave transmitted by the optimal helix [18].

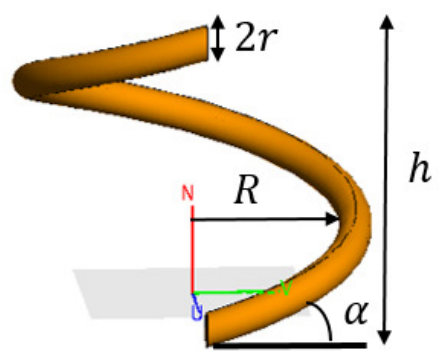

Fig. 1. The chiral MTM inclusion.

Fig. 1 shows the structure of the optimal helix that will be studied using CMA and EEM. The design of the structure consists in a single turn PEC strip helix whose dimensions are $\mathrm{R}=7.75 \mathrm{~mm}, h=12 \mathrm{~mm}, 2 r=1.55 \mathrm{~mm}$ with a pitch angle of $\alpha=13.65^{\circ}$ [18].

\section{REsults}

\section{A. Modal Analysis of the Chiral MTM}

The MoM solver of the commercial tool FEKO is used to design the chiral helix described in section III and calculate its impedance matrix $Z$. The $Z$-matrix is extracted and the CMs and eigenmodes are computed. For EEM, the eigen modes are sorted according to the ratio between the imaginary and real parts of the complex eigenvalues. The chosen frequency band is relatively narrow and no modal tracking issues were observed for CMA and EEM for the first two modes.

The different modal metrics of CMA and EEM are analysed and discussed showing how the properties of chiral MTMs can be highlighted using these metrics. The modal approach differs from [18], [30] where reflection and transmission coefficients, either numerically or analytically determined, are used for the analysis of the chiral MTMs.
1) Modal net stored energy: The modal net energy stored $\Re e\left(W_{\mathrm{m}}-W_{\mathrm{e}}\right)$ by the eigenmodes and CMs of the chiral helix are evaluated and compared. The first two modes are calculated for both modal methods assuming that only two modes are sufficient to analyze electrically small structures [31]. $\Re e\left(W_{m}-W_{e}\right)$ are plotted in Fig. 2 for CMA and EEM.
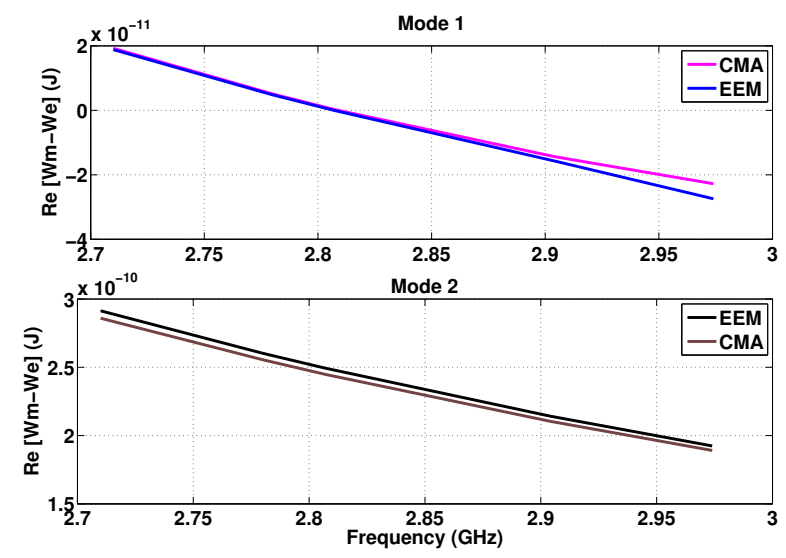

Fig. 2. Real-part of the net stored energy against frequency for the modes 1 and 2 computed by CMA and EEM.

For modes 1 and 2, the net stored energy (normalised to the radiated power) is similar for CMA and EEM. A resonance is identified when $\Re e\left(W_{m}-W_{e}\right)$ is zero. For both CMA and EEM, the helix resonates at $2.82 \mathrm{GHz}$. The net stored energy of mode 1 is positive before the resonant frequency and negative after the resonant frequency, showing that the energy switches from magnetic to electric at resonance. Mode 2 presents a net stored magnetic energy greater than the electric one in the whole frequency band of interest for both methods. It is found that the eigenmodes and the CMs behave similarly in terms of net stored energy.

2) Modal Significance $(M S)$ : In CMA and EEM theories, the MS is an important parameter as it states the coupling capabilities of each mode to the external source. As described in (12), the MS transforms the eigenvalues interval from $[-\infty,+\infty]$ to $[0,1]$. Fig. 3 shows the MS evaluated by CMA and EEM.

Only mode 1 is a resonating mode since it has MS $>0.7$ [14] for both CMA and EEM. The resonant frequency is $2.82 \mathrm{GHz}$. The MS of mode 2 and 3 is less than 0.7 in the whole frequency band. The MS of the CMs is similar to the MS of the eigenmodes for the chiral helix.

3) Modal surface current distribution: To provide a better analysis of the eigenmodes and CMs, it is essential to study the current distribution of the different modes. Fig. 4 presents the modal current of the first two modes for both methods.

For CMA, the current are real quantities, as demonstrated in section II. Therefore, the characteristic currents are equiphase. For EEM, the eigenmodes are complex and the current distribution is not equiphase (phase not shown). For both methods, only mode 1 can radiate since it is a resonating mode. Unlike mode 2, the current is asymmetric and this will cause a cancellation of the electric field $\vec{E}$ in the far field region. The other modes can hardly be excited at $2.82 \mathrm{GHz}$, since their 


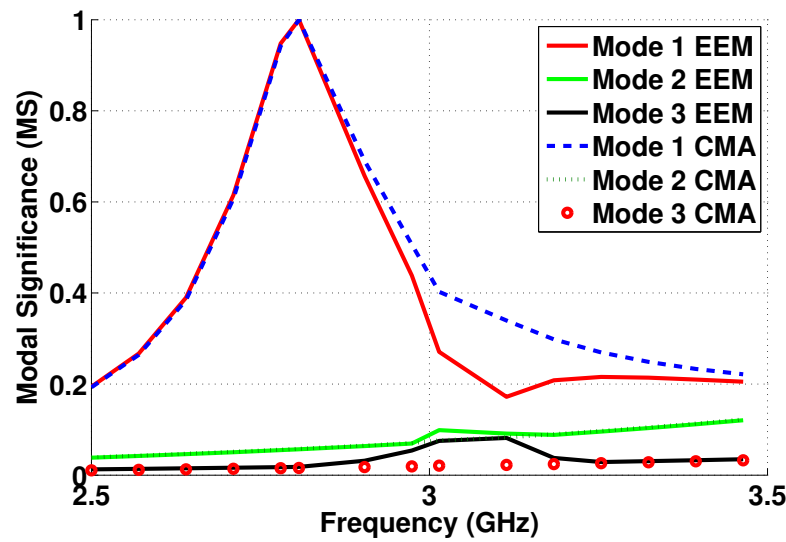

Fig. 3. Modal significance of the first three modes using CMA and EEM against frequency.

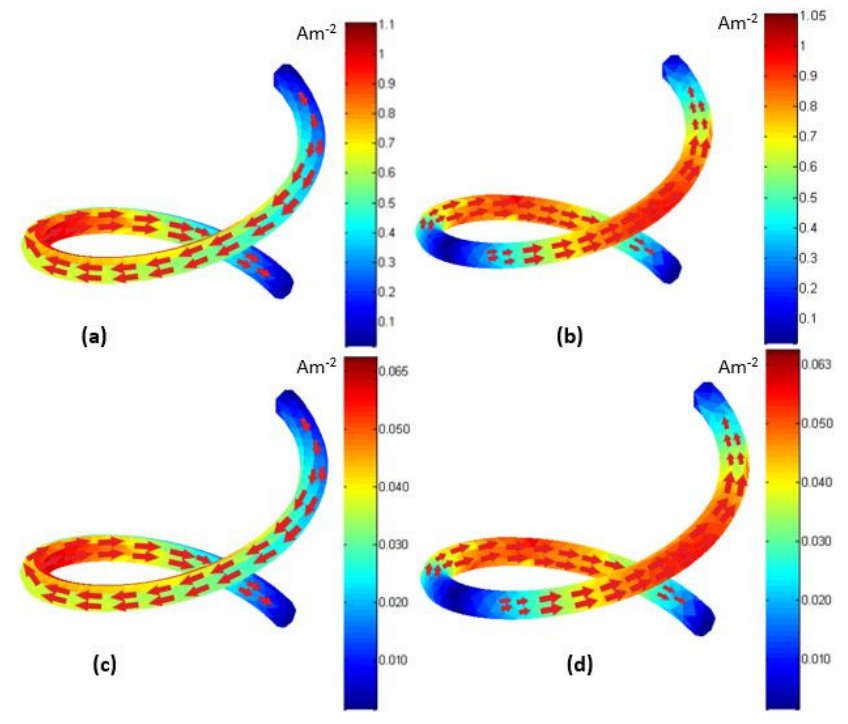

Fig. 4. Magnitude of the surface Current for the first two modes for CMA and $\mathrm{EEM}$ at $2.8 \mathrm{GHz}$ (isometric view) (a) Current distribution of mode 1 by CMA with maximum of $1.1 \mathrm{Am}^{-2}$ (b) Current distribution of mode 2 by CMA with maximum of $1.05 \mathrm{Am}^{-2}$ (c) Current distribution of mode 1 by EEM with maximum of $0.065 \mathrm{Am}^{-2}$ (d) Current distribution of mode 2 by EEM with maximum of $0.063 \mathrm{Am}^{-2}$.

MS is pretty low. The two methods behave similarly in terms of current distribution but they show differences in terms of magnitude (for mode 1 , the maximum value is $1.1 \mathrm{Am}^{-} 2$ for CMA and $0.065 \mathrm{Am}^{-} 2$ for EEM).

4) Modal Weighting Coefficient $(M W C)$ : In this part, the asymmetric transmission property of the chiral helix is analyzed using MWCs, the figure of merit characterizing how each mode reacts under an external excitation. The excitation is provided by a linearly-polarized plane wave propagating in the forward or backward directions at an incidence angle of $45^{\circ}$ The two possible configurations of polarization TE and TM are depicted in Fig. 5.

In the TE case, the electric field $\vec{E}$ is polarized along the helix axis ( $\vec{x}$ axis) whereas the magnetic field $\vec{H}$ is along the helix axis in the TM case. The excitation source is placed at

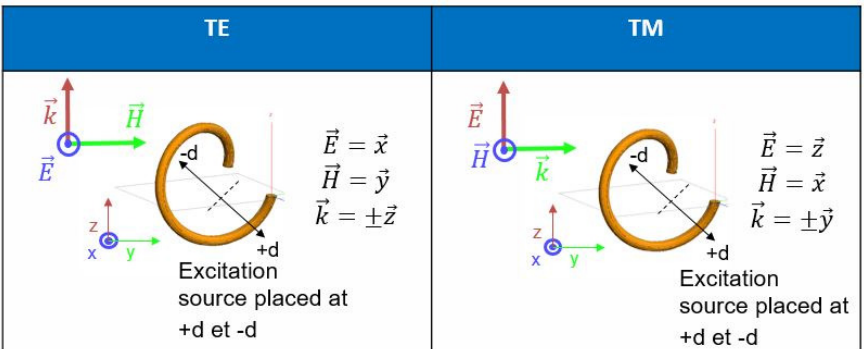

Fig. 5. TE and TM polarization with an incidence angle $45^{\circ}$.

$\pm d$ in the $\vec{x}$ direction to analyze the two opposite directions of propagation. Then, the MWC of the dominant mode, i.e., mode 1 , is computed for the forward and backward propagation. Fig. 6. shows the MWC of mode 1 under TE and TM polarizations.

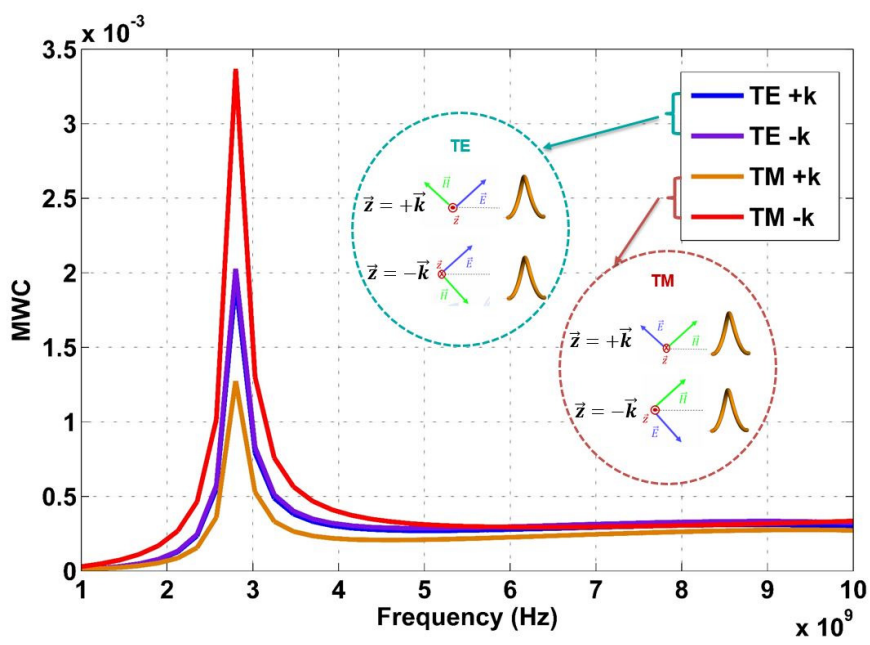

Fig. 6. Modal Weighting coefficient of Mode 1 under TE and TM with an incidence angle $45^{\circ}$. Inset depicts a projection of the helix in the $x y$ plane.

It is clear from Fig. 6 that an asymmetric transmission is observed since the response of the chiral helix is different for forward and backward propagation. The difference between the two responses is very large in the TM case and much lower in the TE case. We conclude that the helix response is more affected by a magnetic excitation as the net stored energy in the near region of the helix is electric as demonstrated above.

To sum up the previous results, it has been demonstrated that the different metrics evaluated by both CMA and EEM are similar in terms of stored energy, MS and modal current distribution. Therefore chiral helices can be analyzed using either CMA or EEM. In addition, MWC is a good metric to highlight chiral properties in modal techniques.

\section{B. Full Wave Simulation of the Chiral MTM}

In this section, the properties of the chiral MTM are analysed in terms of cross polarization conversion using the scattering parameters of an array of periodically distributed chiral helices with a periodicity of $15 \mathrm{~mm}$ and $20 \mathrm{~mm}$ along the $\vec{y}$ and $\vec{x}$ axes respectively. The periodic array is 
investigated with the frequency domain finite-element solver of the commercial simulation tool ANSYS HFSS. FloquetBloch periodic boundary conditions are applied. Floquet ports are used for the excitation and an incidence angle of $45^{\circ}$ is applied. Only the fundamental mode is considered in each configuration.

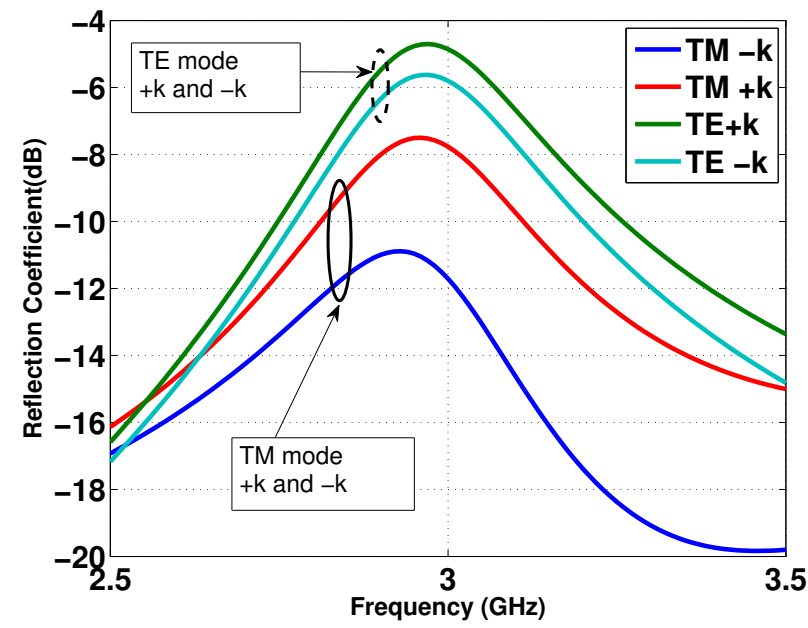

Fig. 7. Reflection coefficient of the chiral helix for the TE and TM polarization with an incidence angle of $45^{\circ}$ for different propagation directions.

The reflection coefficients are presented Fig. 7 for the TE and TM configurations and the forward and backward propagation directions with a $45^{\circ}$ incidence angle. The resonant frequency is at $2.82 \mathrm{GHz}$. For the TE configuration, the reflection coefficients for the two opposite propagation directions show a slight difference of $1 \mathrm{~dB}$ while the difference is more significant of $4 \mathrm{~dB}$ for the TM configuration. This highlights that the full-wave simulations validate the asymmetric transmission of chiral helices previously demonstrated by modal analysis (MWCs).

\section{Discussions}

The results presented in section IV-A-4 has shown that Modal Weighting Coefficient is good metric to demonstrate chiral properties. This has been further validated by the fullwave analysis presented in IV-B. Indeed for the chiral MTM (Fig. 1), the asymmetric transmission is slightly different for the TE forward and backward propagation. For the TM configuration, the difference on the reflection coefficients is more remarkable for the two opposite propagation directions. A good agreement between the modal analysis and the full wave simulation results is found. To provide further comparison between the two modal techniques (EEM and CMA), the surface current density on the chiral MTM is calculated. Fig. 8 shows the current given by the full wave analysis and modal approaches for mode 1 by CMA and EEM.

Mode 1 being resonant and predominant, it is the only mode shown here. The maximum values of the magnitude of the surface current density are given in Table. III.
TABLE III

COMPARISON BETWEEN THE MAXIMUM VALUES OF THE SURFACE CURRENT DENSITY COMPUTED BY FULL-WAVE ANALYSIS, CMA AND EEM.

\begin{tabular}{|l|l|l|}
\hline $\begin{array}{l}\text { Full-wave analy- } \\
\text { sis }\end{array}$ & CMA (Mode 1) & EEM (Mode 1) \\
\hline $0.0721 \mathrm{Am}^{-2}$ & $1.1027 \mathrm{Am}^{-2}$ & $0.0662 \mathrm{Am}^{-2}$ \\
\hline
\end{tabular}

Though, from a qualitative point of view, the current distribution is identical in all approaches and from a quantitative point of view, EEM shows closer results to the full wave analysis. To conclude, the results given by CMA and EEM show similarities in terms of net stored energy, current distribution and modal significance, therefore chiral MTMs can be analyzed using CMA or EEM. Moreover, modal approaches can be used to demonstrate the asymmetric transmission of chiral MTMs.

\section{CONCLUSION}

This paper described the analysis of a complex MTM, i.e. a chiral MTM inclusion based on a spiral helix using two modal analyses, CMA and EEM. The chiral helix with a single turn is characterized by an intrinsic chirality, i.e., a CP wave transmission at normal incidence. The two methods showed similarities in terms of resonant frequency, modal significance, net stored energies and surface current density distribution. Similar current distributions were observed for both modal methods and the full-wave analysis one. However, current values (maximum of current at resonance) are much closer to the direct analysis for EEM.

To demonstrate that chiral MTMs exhibit an asymmetric transmission for linear polarization, the MWCs were evaluated for TE and TM configurations of forward and backward propagation directions at an incidence angle of $45^{\circ}$. Different MWCs were observed for the two propagation directions which was validated and confirmed using the full wave simulation.

The effect of the chiral MTM helix on the field polarization can be exploited for the design of circularly polarized MTMinspired antennas. The use of modal approaches and more specifically EEM should prove to be useful to take into account the coupling between the chiral MTMs and the antenna.

\section{ACKNOWLEDGMENT}

The authors acknowledge funding from I-Site FUTURE in the framework of the Western project. One of the authors acknowledge partial funding by the regional project SMARTIES in the framework of the ELSAT 2020 program co-financed by the European Union with the European Regional development fund, the French state and Hauts de France Regional council.

\section{REFERENCES}

[1] H. Xiong, J.-S. Hong, and Y.-H. Peng, "Impedance bandwidth and gain improvement for microstrip antenna using metamaterials," Radioengineering, vol. 21, no. 4, pp. 993-998, 2012. 

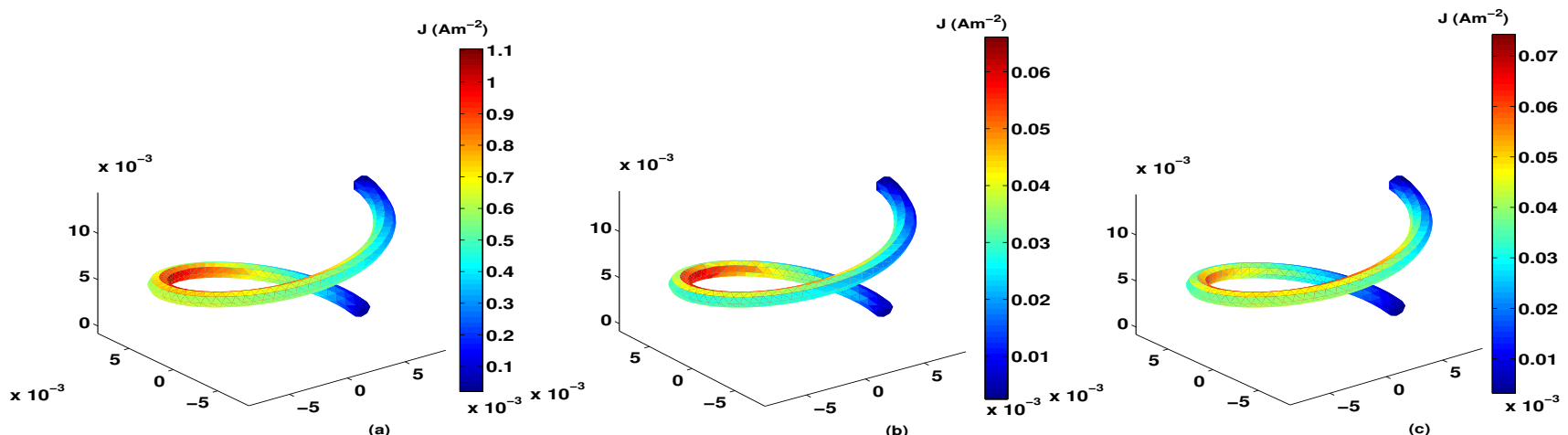

(c)

Fig. 8. (a) Magnitude of the surface current density of mode 1 at $2.82 \mathrm{GHz}$ by CMA (b) Magnitude of the surface current density of mode 1 at $2.82 \mathrm{GHz}$ using EEM (c) Magnitude of the total current density evaluated by full-wave analysis at $2.82 \mathrm{GHz}$ (isometric view).

[2] A. Alu, M. G. Silveirinha, A. Salandrino, and N. Engheta, "Epsilon-nearzero metamaterials and electromagnetic sources: Tailoring the radiation phase pattern," Physical review B, vol. 75, no. 15, p. 155410, 2007.

[3] R. W. Ziolkowski, P. Jin, and C.-C. Lin, "Metamaterial-inspired engineering of antennas," Proceedings of the IEEE, vol. 99, no. 10, pp. 1720-1731, 2010

[4] Z. Li, R. Zhao, T. Koschny, M. Kafesaki, K. B. Alici, E. Colak, H. Caglayan, E. Ozbay, and C. Soukoulis, "Chiral metamaterials with negative refractive index based on four " $\mathrm{u}$ " split ring resonators," Applied Physics Letters, vol. 97, no. 8, p. 081901, 2010.

[5] B. Wang, J. Zhou, T. Koschny, and C. M. Soukoulis, "Nonplanar chiral metamaterials with negative index," Applied Physics Letters, vol. 94, no. 15 , p. $151112,2009$.

[6] S. V. Zhukovsky, A. V. Novitsky, and V. M. Galynsky, "Elliptical dichroism: operating principle of planar chiral metamaterials," Optics letters, vol. 34, no. 13, pp. 1988-1990, 2009.

[7] J. Y. Chin, M. Lu, and T. J. Cui, "Metamaterial polarizers by electricfield-coupled resonators," Applied Physics Letters, vol. 93, no. 25, p. 251903, 2008

[8] T. Li, H. Liu, T. Li, S. Wang, F. Wang, R. Wu, P. Chen, S. Zhu, and $\mathrm{X}$. Zhang, "Magnetic resonance hybridization and optical activity of microwaves in a chiral metamaterial," Applied Physics Letters, vol. 92, no. 13, p. 131111, 2008.

[9] M. H. Rabah, D. Seetharamdoo, and M. Berbineau, "Analysis of miniature metamaterial and magnetodielectric arbitrary-shaped patch antennas using characteristic modes: Evaluation of the $q$ factor," IEEE Trans. Antennas Propagat., vol. 64, no. 7, pp. 2719-2731, 2016.

[10] S. E. Mendhe and Y. P. Kosta, "Metamaterial properties and applications," International Journal of Information Technology and Knowledge Management, vol. 4, no. 1, pp. 85-89, 2011.

[11] R. W. Ziolkowski, "Applications of metamaterials to realize efficient electrically small antennas," in IWAT 2005. IEEE International Workshop on Antenna Technology: Small Antennas and Novel Metamaterials. IEEE, 2005, pp. 7-10.

[12] A. Ramm, "Theoretical and practical aspects of singularity and eigenmode expansion methods," IEEE Trans. Antennas Propagat., vol. 28, no. 6, pp. 897-901, 1980.

[13] M. Vogel, G. Gampala, D. Ludick, U. Jakobus, and C. Reddy, "Characteristic mode analysis: Putting physics back into simulation," IEEE Antennas Propagat. Mag., vol. 57, no. 2, pp. 307-317, 2015.

[14] Y. Chen and C.-F. Wang, Characteristic modes: Theory and applications in antenna engineering. John Wiley \& Sons, 2015.

[15] M. Capek, L. Jelinek, P. Hazdra, and J. Eichler, "The measurable $q$ factor and observable energies of radiating structures," IEEE Trans. Antennas Propagat., vol. 62, no. 1, pp. 311-318, 2013.

[16] C. Ozuem, D. Seetharamdoo, M. H. Rabah, and H. Sakli, "Stored energy evaluation of metamaterials based on theory of characteristic mode," in 12th European Conference on Antennas and Propagation (EuCAP 2018). IEEE, 2018, pp. 1-5.
[17] I. Semchenko, A. Balmakou, S. Khakhomov, and S. Tretyakov, "Stored and absorbed energy of fields in lossy chiral single-component metamaterials," Physical Review B, vol. 97, no. 1, p. 014432, 2018.

[18] E. Saenz, I. Semchenko, S. Khakhomov, K. Guven, R. Gonzalo, E. Ozbay, and S. Tretyakov, "Modeling of spirals with equal dielectric, magnetic, and chiral susceptibilities," Electromagnetics, vol. 28, no. 7, pp. 476-493, 2008.

[19] N. Kari, C. Ozuem, D. Seetharamdoo, J.-M. Laheurte, and F. Sarrazin, "Analysis of a chiral helix metamaterial using eigenmode expansion method and characteristic mode theory," in 2019 IEEE International Symposium on Antennas and Propagation and USNC-URSI Radio Science Meeting. IEEE, 2019, pp. 491-492.

[20] R. F. Harrington and J. R. Mautz, Theory and computation of characteristic modes for conducting bodies. Electrical Engineering Department, Syracuse University, 1970.

[21] R. F. Harrington, Field computation by moment methods. Wiley-IEEE Press, 1993.

[22] T. Bernabeu-Jiménez, A. Valero-Nogueira, F. Vico-Bondia, and A. A. Kishk, "A comparison between natural resonances and characteristic mode resonances of an infinite circular cylinder," IEEE Trans. Antennas Propagat., vol. 65, no. 5, pp. 2759-2763, 2017.

[23] R. Garbacz, "A general expansion for radiated and scattered fields," $\mathrm{Ph}$.D. dissertation, Ph. D. Dissertation, Ohio State University, Columbus, Ohio, 1968.

[24] S. Huang, J. Pan, and Y. Luo, "Study on the relationships between eigenmodes, natural modes, and characteristic modes of perfectly electric conducting bodies," International Journal of Antennas and Propagation, vol. 2018, 2018.

[25] K. F. Warnick, Numerical analysis for electromagnetic integral equations. Artech House, 2008.

[26] G. A. Vandenbosch, "Reactive energies, impedance, and $q$ factor of radiating structures," IEEE Trans. Antennas Propagat., vol. 58, no. 4, pp. 1112-1127, 2010.

[27] M. Capek and L. Jelinek, "Optimal composition of modal currents for minimal quality factor q," IEEE Trans. Antennas Propagat., vol. 64, no. 12 , pp. $5230-5242,2016$.

[28] X. Ma, M. Pu, X. Li, Y. Guo, P. Gao, and X. Luo, "Meta-chirality: Fundamentals, construction and applications," Nanomaterials, vol. 7, no. 5, p. 116, 2017.

[29] C. Huang, Y. Feng, J. Zhao, Z. Wang, and T. Jiang, "Asymmetric electromagnetic wave transmission of linear polarization via polarization conversion through chiral metamaterial structures," Physical Review B, vol. 85, no. 19, p. 195131, 2012.

[30] Y. Z. Cheng, Y. Nie, Z. Z. Cheng, X. Wang, and R. Z. Gong, "Asymmetric chiral metamaterial circular polarizer based on twisted split-ring resonator," Applied Physics B, vol. 116, no. 1, pp. 129-134, 2014.

[31] M. Cabedo-Fabres, E. Antonino-Daviu, A. Valero-Nogueira, and M. F. Bataller, "The theory of characteristic modes revisited: A contribution 
to the design of antennas for modern applications," IEEE Antennas

Propagat. Mag., vol. 49, no. 5, pp. 52-68, 2007. 